\title{
PREFACE
}

\section{Preface (Vol 37, Issue 2)}

\author{
Nadia Magnenat-Thalmann ${ }^{1}$
}

Accepted: 18 January 2021 / Published online: 1 February 2021

(c) The Author(s), under exclusive licence to Springer-Verlag GmbH, DE part of Springer Nature 2021

In this issue, we have 14 regular papers:

The first paper is by Alberto Sanchez et al., titled:" Feature selection based on star coordinates plots associated with eigenvalue problems"

The second paper is from Hana Ben Fredj et al., titled "Face recognition in unconstrained environment with CNN"

The third paper is from Dominique Beaini et al., titled: "Deep green function convolution for improving saliency in convolutional neural networks"

The fourth paper is from Fazhi He et al., titled: "A multiphase blending method with incremental intensity for training detection networks"

The fifth paper is by Fang Wang et al., titled "A rapid vortex identification method using fully convolutional segmentation network"

The sixth paper is from Ravimal Bandara et al., titled:" Deep learned compact binary descriptor with a lightweight network-in-network architecture for visual description"

The seven paper is from Sanasam Inunganbi et al., titled:" Meitei Mayek handwritten dataset: compilation, segmentation, and character recognition"

The eight paper is from Murat Kurt, et al., titled "GenSSS:" a genetic algorithm for measured subsurface scattering representation"

The nine paper is from Zhengxing Sun et al., titled" A structural-constraint 3D point clouds segmentation adversarial method"
The tenth paper is from Xiaolong Zhang, et al., titled" DART: a visual analytics system for understanding dynamic association rule mining"

The eleven paper is from Jixin Liu et al., titled "Privacy-preserving video fall detection using visual shielding information"

The twelve paper is from Zhiguo Cao et al., titled "Abrupt-motion-aware lightweight visual tracking for unmanned aerial vehicles"

The thirteen paper is from Sajjad Bagheri Baba Ahmadi et al., titled: "An intelligent and blind image watermarking scheme based on hybrid SVD transforms using human visual system characteristics"

The fourteen paper is from Suting Chen et al., titled: "A simplified ICA-based local similarity stereo matching"

Nadia Magnenat Thalmann

Editor-in-Chief

The Visual Computer

Publisher's Note Springer Nature remains neutral with regard to jurisdictional claims in published maps and institutional affiliations.
Nadia Magnenat-Thalmann

thalmann@miralab.ch

1 MIRALab-CUI, University of Geneva, Battelle, Building A,

7, Route de Drize, 1227 Carouge, Geneva, Switzerland 